Note on the Governing of Electromotors

This content has been downloaded from IOPscience. Please scroll down to see the full text. 1887 Proc. Phys. Soc. London 9296

(http://iopscience.iop.org/1478-7814/9/1/342)

View the table of contents for this issue, or go to the journal homepage for more

Download details:

IP Address: 131.91.169.193

This content was downloaded on 03/10/2015 at 19:37

Please note that terms and conditions apply. 
Considering the considerable differences in the relations of the currents, the constancy of the angle of lag appears to mo to point to a simple law connecting it with the magnetization.

There was no very accurate speed-indicator employed, and the speed was approximately the same in the first six experiments. In the seventh experiment the speed was purposely much diminished, by about one third of that in the former cases, and in this experiment alone does the lag appear at less than $5^{\circ}$.

\section{Note on the Governing of Electromotors.} By:W. E. Ayrton and Jomn Perry*.

IN 1882 a method was brought forward by us for governing electromotors, so that their speed should not be varied by an alteration in the load. The method, which was worked out theoretically and experimentally, was based on the combination of a motor, which converted electric energy into mechanical energy, with a brake dynamo, which reconverted the surplus mechanical energy into electric energy. After passing through various stages the arrangement finally resolved itself into an electromotor having the field-magnet wound with a shunt- and a series-coil, the coils being so connected with tho rest of the circuit that the currents passed round them in opposite directions. Hence the magnetic excitation of the field-magnet was produced by the difference in the number of ampere-turns of the two circuits passing round it.

This method of governing motors, which has since 1882 been reinvented in France by M. Deprez, in America by Mr. Sprague, and which has formed the subject of an exhaustive series of experiments carried out in Germany by Dr. Fröhlich, answers well and produces a very fair constancy of speed for very considerable variations of the load. But it has a very decided defect, arising from the fact that since the series- and shunt-coils on the field-magnet oppose one another's action, the magnetization cannot be produced as economically as if there were no such differential action. Mainly on this account we have not developed our method

* Read May 26, 1888. 
of governing as energetically as we might otherwise have been led to do.

In our original investigation of the subject wo considered the conditions for governing motors either when a constant PD. (potential-difference) or when a constant current was supplied, and in both cases the practical solution we arrived at was a differential winding of the field-magnet. But we have since noticed that, in the case of the supply conditions being constant current, our original equations could be differently interpreted, and that the demagnetizing series could be dispensed with. Consequently a motor for constant current can be made to go at a constant speed with a varying load, and in addition to have the high efficiency of a wellmade dynamo.

We shall for the general consideration of the problem use the simplest equations for a motor, disrogarding saturation, as we did in our original paper, since, although the results so obtained for critical speed, \&c., cannot bo used directly without correction in actual practice, these equations are sufficiently accurate to show whother or not a certain combination is a possible or an impossible one for obtaining a certain required result.

If $\mathrm{E}$ be the back E.M.F. in the armature of a motor at a speed of $n$ revolutions, if $p$ be a term depending on the permanent magnetism in the iron of the field-magnet, $S$ the current passing round the series-coil, and $Z$ the current round the shunt-coil, we have

$$
\mathbf{E}=n(p \pm q \mathbf{S} \pm t \mathrm{Z}) . \quad \cdot \quad \cdot \quad \cdot .
$$

The signs + or - in the two terms being used as the seriesand the shunt-currents respectively help or opposo the permanent magnetism.

If $s$ and $z$ be the resistances of the series- and shunt-coils respectively, $a$ the resistance of the armature, and $A$ the current round the armature, we have in the case of what has been called a short shunt (fig. 1),

$$
\mathrm{E}=\mathrm{Z} z-\mathrm{A} a, \cdot \cdot \cdot \cdot \cdot \cdot .
$$

and in the case of what has been called a long shunt (fig. 2 ),

vox IX.

$$
\mathrm{E}=\mathrm{Z} z-\mathrm{A}(a+s) \text {; }
$$


and in both cases, if $\mathrm{C}$ be the constant current supplied to the motor,

$$
\mathrm{C}=\mathrm{A}+\mathrm{Z} \cdot \cdot \cdot \cdot \cdot \cdot \cdot \cdot \cdot \cdot \cdot
$$

Fig. 1.

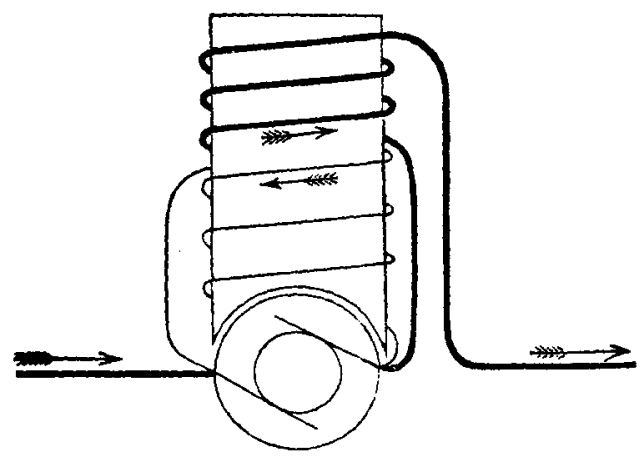

Fig. 2.

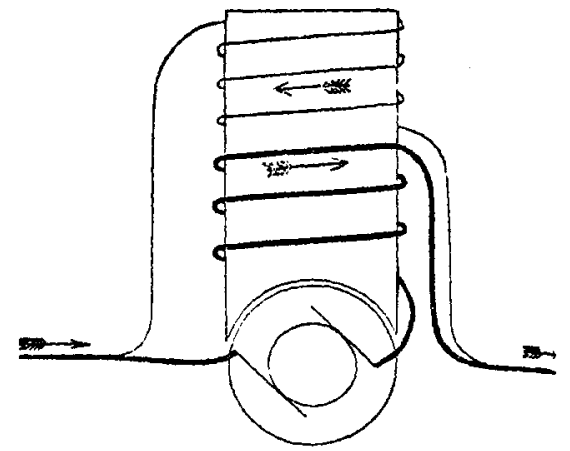

Now, remembering that in the case of a short shunt (fig. 1), $\mathrm{C}$ is equal to $\mathrm{S}$, equations (1), (2), and (4) lead to

$$
n\{p \pm q \mathrm{C} \pm t(\mathrm{C}-\mathrm{A})\}=(\mathrm{C}-\mathrm{A}) \approx-\mathrm{A} a ; .
$$

and in the case of a long shunt (fig. 2), remembering that $\mathrm{C}$ is equal to $A$, equations (1), (3), and (4) lead to

$$
n\{p \pm q \mathrm{~A} \pm t(\mathrm{C}-\mathrm{A})\}=(\mathrm{C}-\mathrm{A}) z-\mathrm{A}(a+s) \text {. }
$$

Equations 5 and 6 have then to be satisfied for all values of A for constant values of $n$ and $C$; hence in the case of a short shunt,

$$
\pm n(-t \mathrm{~A})=-\mathrm{A}(z+\alpha), \quad . \quad . \quad . \quad .
$$

and in the case of a long shunt

$$
\pm n(q-t) \mathrm{A}=-\mathrm{A}(z+a+s)
$$

But $t, q, z, a$, and $s$ are all positive quantities, and since $t$ is always larger than $q$, that is the number of turns in the shunt-coil is always larger than the number of turns in the series-coil, it follows that to satisfy either equation (7) for the short shunt or equation ( 8 ) for the long shunt, the positive value of $t$ in the original equation (1) can in either case alone be employed. In other words, whatever be the arrangement of the series-coil, the shunt-coil must be a magnetizing and not a demagnetizing one.

This result we pointed out in our original paper on "Electromotors and their Government," in vol. xii. of the "Journal of the Society of Telegraph Engineers and Elec- 
tricians,' since on page 314 we stated that the solution for constant current was a shunt-motor with a series demagnetizing-coil; and it would be unnecessary to refer to this point again were it not that in one of the standard English treatises in which our method of governing motors is described in detail, the only solution for constunt current that we are credited with is the impossible one of a series-motor with shunt demagnetizing-coil, the author not having observed that such a combination, whether used as a short or as a long shunt, could only make a motor run at a uniform speed when the speed was negative. Now, what does a negative value of $n$ mean? It means that the armature must run the other way, or, in other words, that what was supposed to be a series-motor must in reality be the armature combined with a series demagnetizing-coil, that is must act as a series brakedynamo, and the supposed shunt demagnetizing-coil must, in conjunction with the armature, act as a shunt-motor; or, in other words, the solution is as we originally stated it-a shunt-motor with series demagnetizing-coil.

In another important English work our method is given correctly, but it is stated that the method would not work, because when a greater load was put on the motor the armature would go more slowly, the back F.M.F. would therefore be diminished, and less current would pass round the shunt-coil of the field-magnet. Hence the field-magnet would be weakened, which would cause the motor to go more slowly, and so on until it stopped and possibly reversed. This objection is a curious one, because exactly the same apparent difficulty might have been stated by the author of this treatise when he was dealing with our method of governing motors to run at a constant P.D. a few pages earlier. Here, however, he sees the explanation of the difficulty, and he points out quite correctly that, although it might at first sight appear anomalous to say that, in order to keep a motor running at a fixed speed, it is necessary to weaken the field of the motor when the load on it is increased, the explanation is found in the fact that the power depends not merely on the strength of the field but on the current passing through the armature, and that this latter is much increased when the field is weakened. Hence, on the whole, it is quite right to arrange matters so 
that the field is weakened when the load is increased. $\mathrm{W}_{\mathrm{e}}$ say it is curious then, that while the author has explained the apparent difficulty so clearly when he was dealing with our method for governing motors when the supply, condition was a constant P.D., he does not see that exactly the same explanation clears away the difficulty which he has himself raised regarding our method for governing motors to run when supplied with constant current.

What, however, we particularly wish to point ont in this short note is that when the condition of supply is constant current it is not necessary to have a series demagnetizing-coil at all, and consequently the motor can be made to run at constant speed independently of the load, and in addition to work with high efficiency.

We will first see whether such a solution is possible when the condition of supply is constant P.D. Let the motor be a simple sbunt-motor; then, if as before $\mathrm{E}$ be the back F.M.F., $n$ the speed, $p$ a term depending on the permanent magnetism, and $\mathrm{Z}$ the current round the shunt,

also

$$
\mathrm{E}=n(p+t \mathrm{Z}) ; . \quad \cdot \quad \cdot \quad . \quad \text {. }
$$

if $z$ be the resistance of the shunt-coil, $A$ the current in the armature, and a the resistance of the armature; also

$$
\mathrm{V}=\frac{\mathrm{Z}}{z}
$$

where $\mathrm{V}$ is the constant P.D. maintained at the terminal, of the motor;

$$
\therefore \quad n\left(p+t \frac{\mathrm{V}}{\hat{i}}\right)=\mathrm{V}-\mathrm{A} a . \quad . \quad .
$$

If $n$ is to be constant as well as $V$, the left-hand side of the equation is constant, whereas the right-hand side varies with A the current through the armature, which must alter as the power given out by the motor alters. One solution for making this equation always true independent of the value of $A$ is the well-known one of making a very small. When, however, $a$ is not very small, and we have shown in detail elsewhere why we consider it a mistake to make the resistance. of the armature as small in a motor as in a dynamo, equation (10) cannot be fulfilled for constant values of $n$ and $V$, and 
a variable value of $A$; and in order that the equation may be made true a negative term proportional to A must be introduced on the left-hand side. The simplest way of doing this is to add a series demagnetizing-coil. Then (9) becomes

and (10)

$$
\mathrm{E}=n(p-q \mathrm{~A}+t \mathrm{Z})
$$

$$
n\left(p-q \mathrm{~A}+t \frac{\mathrm{V}}{z}\right)=\mathrm{V}-\mathrm{A}(a+s),
$$

an equation which will be always satisfied for any value of $A$ if

$$
n=\frac{a+s}{q} \text {. }
$$

But when the supply is a constant current $\mathrm{C}$ supplied to the motor, the equation for a simple shunt-motor is

and, therefore,

$$
\begin{aligned}
& \mathrm{E}=n(p+t \mathrm{Z}), \\
& \mathrm{E}=\mathrm{Z} z-\mathrm{A} a, \\
& \mathrm{C}=\mathrm{A}+\mathrm{Z} ;
\end{aligned}
$$

$$
n\{p+t(\mathrm{C}-\mathrm{A})\}=(\mathrm{C}-\mathrm{A}) z-\mathrm{A} a . . .
$$

A now appears $w^{\text {th }}$ a negative sign on both sides of the equation (11), and this equation will always be satisfied for any value of $A$ if

$$
n=\frac{z+a}{t}, \quad . \quad . \quad \cdot \quad \cdot \quad . \quad \text {. }
$$

without there being any necessity for using a series demagnetizing-coil. At first sight, then, it appeurs as if an entirely new solution for governing a motor supplied with a constant current had been arrived at, consisting simply of using a shunt-motor. But on further examination it is seen that if we substitute in (11) the value given in (12) for $n$, we obtain for $\mathrm{C}$ the current for which the motor governs :

$$
\begin{aligned}
\mathrm{C} & =\frac{n p}{z-n t} \\
& =-\frac{n p}{\mathrm{~s}} .
\end{aligned}
$$

But as this is negative it is necessary, in order that the shuntmotor may govern, that the terminals of the motor should be reversed. This will reverse the current round the field-ma gnet 
but, by hypothesis, not the residual magnetism, otherwise $p$ would also become negative. The core of the electromagnet must, therefore, be of steel, so strongly magnetized that the shunt-current does not reverse the polarity, and the permanent magnet and rotating armature together form a brake-dynamo while the shunt-coil and the armature will form the motor. But this is simply one of the solutions (a theoretical one of course and not a practical one) given by us on page 310 of our original paper and illustrated in figure 10 on that page.

The fact, however, still remains that in the case of the supply condition being constant current, equation (11) can be satisfied without the employment of a series demagnetizing. coil; and all that we have to do to in order to make the solution a practical one, that is to enable an ordinary field-magnet with soft iron core to be employed, is to alter the equation so that when the speed equation (12) is satisfied (11) leads to a positive value of the current. This result may be obtained by adding to the right-hand side of equation (11) a positive constant term e numerically larger than $n p$, for in that case, if

$$
\begin{aligned}
& n=\frac{z+a}{t} \\
& \mathrm{C}=\frac{e-n p}{a}
\end{aligned}
$$

for all values of .A. Now such a positive term $e$ will be Fig. 3.

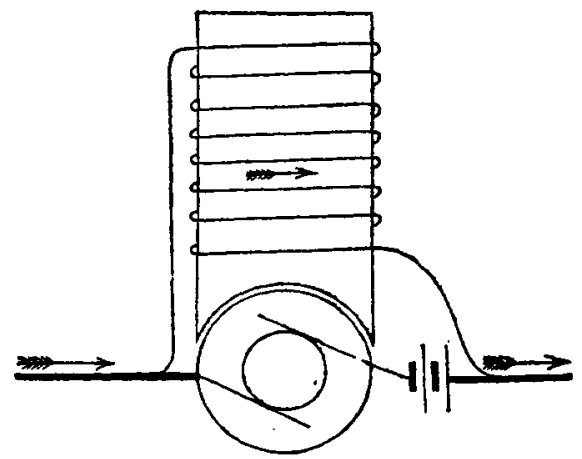

introduced on the right-hand side of equation $e$, if some accumulators of E.M.F. equal to $e$ be introduced into the armature circuit, as in figs 3 , the accumulators being joined uf so as to help the main current $\dot{\mathrm{C}}$. In that case the resistance 
$a$ of the armature must be increased by $a^{\prime}$, the resistance of the accumulators; and the two equations we are finally led to for the speed at which the motor governs, and the constant current for which it governs, are

$$
\begin{aligned}
& n=\frac{z+a+a^{\prime}}{t}, \quad . \quad . \quad . \quad . \quad . . . \\
& \mathrm{C}=\frac{e-n p}{a+a^{\prime}} . \\
& \hline
\end{aligned}
$$

This solution is a practical one, and consists of a simple shunt-motor, with some accumulators in the armature circuit. Further, it is one not requiring many accumulators, for if $n p$, the back E.M.F. of the motor due to the residual magnetism alone be small, as it will be if the field-magnet iron core be soft, and further, if $a+a^{\prime}$ be also not large, e need not be large in order that the value of $\mathrm{C}$, the current for which the motor governs, may be as large as we like. The essence of this new method for making a motor supplied with a constant current revolve at a constant speed independently of the load does not consist in combining a shunt-motor with a series brake-dynamo, which was the essence of our original method, but in combining a shunt-motor with a store of electric energy in the armature-circuit, which store of energy, as the equations show, is automaticilly dealt out to help the motor exactly in proportion as the demand for power is required to keep the speed constant when the load on the motor is varied. And this store can be easily maintained by reversing the connexions of the accumulators when they are disconnected from the motor, and the motor is at rest, and leaving the accumulators without supervision to be charged by the constant current that is continuously supplied to the station.

While on the subject of electromotors it may be well to point out a fact which we thought was well known. viz. that when motors are being tested one against another for efficiency and for power developed per pound weight. it is most important that the motors should each be suppiiea wirn the P.D. they are intended to work at and should be run at their normal speed. But we have been much astonished to find in the 'Technology Quarterly' for September last, published by the Massachusetts Institute of Technology, a 
paper by Mr. H. Clifford on "The Efficiency of Small Electro. Motors," in which the results of experiments on the relative power and efficiency of a number of motors are given without any attention having been paid to the particular P.D. and speed at which each motor was wound to work at. $\mathrm{Mr}$. Clifford's results are arranged in tables which would be of considerable practical value were it not that the essential condition for making such tests has been absolutely disregarded. Hence Mr. Clifford's tables are not only valueless but are most misleading. We are able to speak definitely on the subject, because the results of the tests made on a motor of ours, which are quoted, are made with the motor running at less than half its normal speed, and supplied with less than one quarter of its normal power. As well might incandescent lamps intended to be run with very different P.Ds. be com. pared when run with totally wrong P.Ds., and a 100-volt lamp condemned as useless because when supplied with only 50 volts it emitted but little light.

XLIII. On the Formulee of Bernoulli and of Haecker for the Lifting-power of Magnets. By Professor Silvanus P. THOMPson*.

Fonmules for the lifting-power of magnets have been given by $D$. Bernoulli and by Haecker, and more recently by Ván der Willigen.

Bernoulli's empirical rule $f$ is that the lifting-power varies as the cube root of the square of the magnet's weight, or

$$
\mathrm{P} \propto \sqrt[3]{W^{2}}
$$

where $\mathrm{P}$ is the lifting power or pull exerted by the nagnet in contact with its keeper, and $\mathrm{W}$ the mass of the magnet.

Haecker $\ddagger$ gave precision to the rule by introducing a co. efficient, the numerical value of which varies with the qualities of steel employed. Writing

$$
\mathrm{P}=a \sqrt[3]{\mathrm{W}^{2}}
$$

he found that (when the unit of mass taken was the Germull

* Read May 26, 1888.

+ Actar Helvetica, iii. p. 2:33 (1758).

$\neq$ Pogr. Anni. Irii. p. 321 (18+2), 